\title{
Lump Solutions and Interaction Solutions for the Dimensionally Reduced Nonlinear Evolution Equation
}

\author{
Baoyong Guo, ${ }^{1,2}$ Huanhe Dong $\mathbb{D}^{2}{ }^{2}$ and Yong Fang $\mathbb{D}^{1,2}$ \\ ${ }^{1}$ College of Electrical Engineering and Automation, Shandong University of Science and Technology, Qingdao, \\ Shandong 266590, China \\ ${ }^{2}$ College of Mathematics and Systems Science, Shandong University of Science and Technology, Qingdao, \\ Shandong 266590, China \\ Correspondence should be addressed to Yong Fang; fangyong@sdust.edu.cn
}

Received 13 August 2019; Revised 23 September 2019; Accepted 4 October 2019; Published 30 October 2019

Academic Editor: Marcelo Messias

Copyright (c) 2019 Baoyong Guo et al. This is an open access article distributed under the Creative Commons Attribution License, which permits unrestricted use, distribution, and reproduction in any medium, provided the original work is properly cited.

In this paper, by means of the Hirota bilinear method, a dimensionally reduced nonlinear evolution equation is investigated. Through its bilinear form, lump solutions are obtained. We construct interaction solutions between lump solutions and one soliton solution by choosing quadratic functions and exponential function. Interaction solutions with the combinations of exponential functions and sine function are also given. Meanwhile, the figures of these solutions are plotted. The dynamical characteristics and properties of obtained solutions are discussed, respectively. The results show that the corresponding physical quantities and properties of nonlinear waves are associated with the values of the parameters.

\section{Introduction}

Nonlinear evolution equations (NLEEs) are becoming more and more important in modern science. People have paid much more attention than ever before on the study of NLEEs. They have significant applications in many subject fields, especially in nonlinear science, for instance, mathematical physics, nonlinear mechanics, particle physics, marine science, atmospheric science, and automation. This trend stems from the fact that NLEEs can explain a lot of natural phenomena; for example, in mathematical physics, many physical quantities of nonlinear waves can be described by the parameters of equations and the solutions of equations can also well interpret the propagation of water waves. In order to obtain the solutions of NLEEs, researchers have put forward many methods, including the Hirota direct method [1], Painlevé analysis method [2], inverse scattering transformation (IST) [3, 4], Riemann-Hilbert method [5-7], Lie symmetry method, and so on [8-12]. Among these methods, the Hirota direct method is so prompt and effective. Based on this approach, researchers have obtained many different kinds of solutions, including lump solutions [13-15], breather solutions [16-18], rogue wave solutions [19-21], interaction solutions, and so on [22-26]. With the help of the Riemann-Hilbert method, people also acquire soliton solutions of integrable hierarchies and coupled systems [27-31]. By taking the long wave limit of soliton solutions, rational solutions of NLEEs are presented [32]. Meanwhile, some difference equations also possess lump solutions and interaction solutions, such as the Toda lattice equation [33]. In recent years, researchers generalize the existing NLEEs to new ones and obtain the corresponding lump solutions $[34,35]$. These results are good supplements to the theory of exact solutions for NLEEs.

In this paper, we focus on the $(3+1)$-dimensional nonlinear evolution equation [36]; its form is

$$
3 u_{x z}-\left(2 u_{t}-2 u u_{x}+2 u_{x x x}\right)_{y}+2\left(u_{x} \int_{x} u_{y}\right)_{x}=0 \text {, }
$$

where $u=u(x, y, z, t), \int_{x}$ is the integral with respect to $x$.

Let $z=y$; we derive dimensionally reduced situation of equation (1) as follows: 


$$
3 u_{x y}-\left(2 u_{t}-2 u u_{x}+2 u_{x x x}\right)_{y}+2\left(u_{x} \int_{x} u_{y}\right)_{x}=0
$$

Equation (2) has wide applications in different areas, for example, mathematical physics, ocean science, engineering, and others. It could describe propagation of shallow water wave in nonlinear dispersive channel. So, it is very important to find the exact solution for this dimensionally reduced nonlinear evolution equation.

The structure of this paper is as follows. In Section 2, we present the Hirota bilinear form and lump solutions of equation (2). In Section 3, we obtain interaction solutions with the combination of lump solutions and one soliton solution. In Section 4, we acquire interaction solutions with the combination of two exponential functions and one sine function. In Section 5, some conclusions are given.

\section{Hirota Bilinear Form and Lump Solutions}

2.1. Hirota Bilinear Form of the Dimensionally Reduced Nonlinear Evolution Equation. By means of variable transformation $u=-6(\ln F)_{x x}$, we transform equation (2) into the Hirota bilinear form $[37,38]$ :

$$
\left(2 D_{y} D_{t}+2 D_{x}^{3} D_{y}-3 D_{x} D_{y}\right) F \cdot F=0,
$$

where $D$ is the Hirota bilinear differential operator which is defined as follows:

$$
\begin{aligned}
& D_{x}^{m} D_{y}^{n} D_{t}^{l} F \cdot G \\
& \quad=\left.\left(\frac{\partial}{\partial x}-\frac{\partial}{\partial x^{\prime}}\right)^{m}\left(\frac{\partial}{\partial y}-\frac{\partial}{\partial y^{\prime}}\right)^{n}\left(\frac{\partial}{\partial t}-\frac{\partial}{\partial t^{\prime}}\right)^{l} F(x, y, t) G\left(x^{\prime}, y^{\prime}, t^{\prime}\right)\right|_{x^{\prime}=x, y^{\prime}=y, t^{\prime}=t} .
\end{aligned}
$$

2.2. Lump Solutions Consisting of Two Quadratic Functions. In order to obtain lump solutions of equation (2), we take function $F$ in the following form:

$$
\begin{aligned}
F & =f_{1}^{2}+f_{2}^{2}+\alpha_{9}, \\
f_{1} & =\alpha_{1} x+\alpha_{2} y+\alpha_{3} t+\alpha_{4}, \\
f_{2} & =\alpha_{5} x+\alpha_{6} y+\alpha_{7} t+\alpha_{8},
\end{aligned}
$$

where $\alpha_{i}(1 \leq i \leq 9)$ are real parameters to be determined.

Substituting equation (5) into equation (3) and considering the coefficients of all powers of the variables to be 0 , with the help of Maple, we have the following relations:

$$
\begin{aligned}
& \alpha_{3}=\frac{3}{2} \alpha_{1}, \\
& \alpha_{6}=-\frac{\alpha_{1} \alpha_{2}}{\alpha_{5}}, \\
& \alpha_{7}=\frac{3}{2} \alpha_{5},
\end{aligned}
$$

where $\alpha_{1}, \alpha_{2}, \alpha_{4}, \alpha_{5}, \alpha_{8}$, and $\alpha_{9}$ are free parameters.

For convenience, we let the parameters to be

$$
\begin{aligned}
& \alpha_{1}=1, \\
& \alpha_{2}=1, \\
& \alpha_{4}=0, \\
& \alpha_{5}=1, \\
& \alpha_{8}=0, \\
& \alpha_{9}=1 .
\end{aligned}
$$

Consequently, the lump solutions of equation (2) can be written as

$$
\begin{aligned}
u= & -6(\ln F)_{x x}=\left(-6\left(\left[\left[2\left(\alpha_{1}^{2}+\alpha_{5}^{2}\right)\left(\left(\alpha_{1} x+\alpha_{2} y+(3 / 2) \alpha_{1} t+\alpha_{4}\right)^{2}+\left(\alpha_{5} x-\left(\alpha_{1} \alpha_{2} / \alpha_{5}\right) y+(3 / 2) \alpha_{5} t+\alpha_{8}\right)^{2}+\alpha_{9}\right)\right]\right.\right.\right. \\
& \left.\left.-\left[2\left(\alpha_{1}\left(\alpha_{1} x+\alpha_{2} y+(3 / 2) \alpha_{1} t+\alpha_{4}\right)+\alpha_{5}\left(\alpha_{5} x-\left(\alpha_{1} \alpha_{2} / \alpha_{5}\right) y+(3 / 2) \alpha_{5} t+\alpha_{8}\right)\right)\right]^{2}\right]\right) \\
& \cdot\left(\left[\left(\alpha_{1} x+\alpha_{2} y+(3 / 2) \alpha_{1} t+\alpha_{4}\right)^{2}+\left(\alpha_{5} x-\left(\alpha_{1} \alpha_{2} / \alpha_{5}\right) y+(3 / 2) \alpha_{5} t+\alpha_{8}\right)^{2}+\alpha_{9}\right]^{2}\right) \\
= & -6\left(\left(4(x+y+(3 / 2) t)^{2}+4(x-y+(3 / 2) t)^{2}-(4 x+6 t)^{2}+4\right) \cdot\left(\left[(x+y+(3 / 2) t)^{2}+(x-y+(2 / 3) t)^{2}+1\right]^{2}\right)^{-1}\right)
\end{aligned}
$$


From equations (5) and (6), we can find that the lump solution is a kind of rational solution. Based on parameter relations (6), we know $F$ is analytical if and only if $\alpha_{5} \neq 0$ and $\alpha_{9}>0$. Let us make a simple dynamical characteristic analysis. Figure 1(a) shows the lump solutions of equation (2). When $\alpha_{4}=0, \alpha_{8}=0$, and $t=0$, the lump wave will center at the origin; here we take $\alpha_{1}=1, \alpha_{2}=1$, $\alpha_{4}=0, \alpha_{5}=1, \alpha_{8}=0, \alpha_{9}=1$, and $t=1$. The lump wave is located in arbitrary directions in the space, so it is a kind of localized wave actually. From equation (8), we can find that if $x \longrightarrow \pm \infty$ or $y \longrightarrow \pm \infty$, then $f_{1}$ and $f_{2} \longrightarrow \infty$, $u \longrightarrow 0$. The lump wave has one peak and two valleys, and valleys are symmetrically distributed on both sides of the peak. According to the extreme value principle of multivariate functions, by the calculation, we obtain that the lump wave's minimum point (extreme value point) is $\left(-\left(3\left(\alpha_{1}^{2}+\alpha_{5}^{2}\right) t+2\left(\alpha_{1} \alpha_{4}+\alpha_{5} \alpha_{8}\right) / 2\left(\alpha_{1}^{2}+\alpha_{5}^{2}\right)\right), \alpha_{5}\left(\alpha_{1} \alpha_{8}-\right.\right.$ $\left.\left.\alpha_{4} \alpha_{5}\right) / \alpha_{2}\left(\alpha_{1}^{2}+\alpha_{5}^{2}\right)\right)=(-(3 / 2), 0)$ and the corresponding amplitude is $-\left(12\left(\alpha_{1}^{2}+\alpha_{5}^{2}\right) / \alpha_{9}\right)=-24$. It propagates along the line $y=0$ with the velocity of $-(3 / 2)$. This mode of motion is uniform linear motion in physics.

\subsection{Lump Solutions Consisting of Three Quadratic Functions.}

We will seek for the lump solutions consisting of three quadratic functions. This situation has rarely been seen in existing literatures $[13-15,35]$. In order to do it, we take function $F$ in the following form:

$$
\begin{aligned}
F & =f_{1}^{2}+f_{2}^{2}+f_{3}^{2}+\alpha_{13}, \\
f_{1} & =\alpha_{1} x+\alpha_{2} y+\alpha_{3} t+\alpha_{4}, \\
f_{2} & =\alpha_{5} x+\alpha_{6} y+\alpha_{7} t+\alpha_{8}, \\
f_{3} & =\alpha_{9} x+\alpha_{10} y+\alpha_{11} t+\alpha_{12},
\end{aligned}
$$

where $\alpha_{i}(1 \leq i \leq 13)$ are real parameters to be determined.
Substituting equation (9) into equation (3) and considering the coefficients of all powers of the variables to be 0 , with the help of Maple, we have the following relations:

$$
\begin{aligned}
& \alpha_{1}=-\frac{1}{3} \frac{2 \alpha_{6} \alpha_{7}+3 \alpha_{9} \alpha_{10}}{\alpha_{2}}, \\
& \alpha_{3}=-\frac{1}{2} \frac{2 \alpha_{6} \alpha_{7}+3 \alpha_{9} \alpha_{10}}{\alpha_{2}}, \\
& \alpha_{5}=\frac{2}{3} \alpha_{7}, \\
& \alpha_{11}=\frac{3}{2} \alpha_{9},
\end{aligned}
$$

where $\alpha_{2}, \alpha_{4}, \alpha_{6}, \alpha_{7}, \alpha_{8}, \alpha_{9}, \alpha_{10}, \alpha_{12}$, and $\alpha_{13}$ are free parameters.

For convenience, we let the parameters to be

$$
\begin{aligned}
\alpha_{2} & =1, \\
\alpha_{4} & =0, \\
\alpha_{6} & =1, \\
\alpha_{7} & =1, \\
\alpha_{8} & =0, \\
\alpha_{9} & =1, \\
\alpha_{10} & =1, \\
\alpha_{12} & =0, \\
\alpha_{13} & =1 .
\end{aligned}
$$

Consequently, the lump solutions consisting of three quadratic functions of equation (2) can be written as

$$
\begin{aligned}
u & =-6(\ln F)_{x x} \\
& =-6 \frac{(76 / 9)(-(5 / 3) x+y-(5 / 2) t)^{2}+(76 / 9)((2 / 3) x+y+t)^{2}+(76 / 9)(x+y+(3 / 2) t)^{2}-((76 / 9) x+(38 / 3) t)^{2}+(76 / 9)}{\left[(-(5 / 3) x+y-(5 / 2) t)^{2}+((2 / 3) x+y+t)^{2}+(x+y+(3 / 2) t)^{2}+1\right]^{2}} .
\end{aligned}
$$

Based on parameter relations (10), we know $F$ is analytical if and only if $\alpha_{2} \neq 0$ and $\alpha_{13}>0$. Figure 2(a) shows the lump solutions consisting of three quadratic functions of equation (2). Similar to the previous dynamical characteristic analysis, when $\alpha_{4}=0, \alpha_{8}=0, \alpha_{12}=0$, and $t=0$, the lump wave will center at the origin; here we take $\alpha_{2}=1, \alpha_{4}=$ $0, \alpha_{6}=1, \alpha_{7}=1, \alpha_{8}=0, \alpha_{9}=1, \alpha_{10}=1, \alpha_{12}=0, \alpha_{13}=1$, and $t=1$. From equation (12), we can find that if $x \longrightarrow \pm \infty$ or $y \longrightarrow \pm \infty$, then $f_{1}, f_{2}$, and $f_{3} \longrightarrow \infty, u \longrightarrow 0$. The lump wave consisting of three quadratic functions also has one peak and two valleys, and valleys are symmetrically distributed on both sides of the peak. Similarly, we obtain that the lump wave's minimum point (extreme value point) is $\left(-\left(\left(3\left[\left(4 \alpha_{7}^{2}\left(\alpha_{2}^{2}+\alpha_{6}^{2}\right)+9 \alpha_{9}^{2}\left(\alpha_{2}^{2}+\alpha_{10}^{2}\right)+12 \alpha_{6} \alpha_{7} \alpha_{9} \alpha_{10}\right) t+4 \alpha_{2}\right.\right.\right.\right.$ $\left.\left.\alpha_{7}\left(\alpha_{2} \alpha_{8}-\alpha_{4} \alpha_{6}\right)+6 \alpha_{2} \alpha_{9}\left(\alpha_{2} \alpha_{12}-\alpha_{4} \alpha_{10}\right)\right]\right) / 2\left[4 \alpha_{7}^{2}\left(\alpha_{2}^{2}+\alpha_{6}^{2}\right)+\right.$ $\left.\left.9 \alpha_{9}^{2}\left(\alpha_{2}^{2}+\alpha_{10}^{2}\right)+12 \alpha_{6} \alpha_{7} \alpha_{9} \alpha_{10}\right]\right),-\left(\left(\alpha_{2} \alpha_{4}+\alpha_{6} \alpha_{8}+\alpha_{10} \alpha_{12}\right) /\right.$ $\left.\left.\left(\alpha_{2}^{2}+\alpha_{6}^{2}+\alpha_{10}^{2}\right)\right)\right)=(-(3 / 2), 0)$ and the corresponding amplitude is $-\left(\left(4\left(2 \alpha_{6} \alpha_{7}+3 \alpha_{9} \alpha_{10}\right)^{2} / 3 \alpha_{2}^{2}\right)+\left((16 / 3) \alpha_{7}^{2}+12\right.\right.$ $\left.\left.\alpha_{9}^{2}\right)\right) / \alpha_{13}=-(152 / 3)$. It propagates along the line $y=0$ with the velocity of $-(3 / 2)$. This mode of motion is also uniform linear motion in physics.

Compared with the previous results (Section 2.2), when $\alpha_{4}=0, \alpha_{8}=0$, and $\alpha_{12}=0$, we realize that lump solutions (8) and lump solutions (12) have the same minimum point 


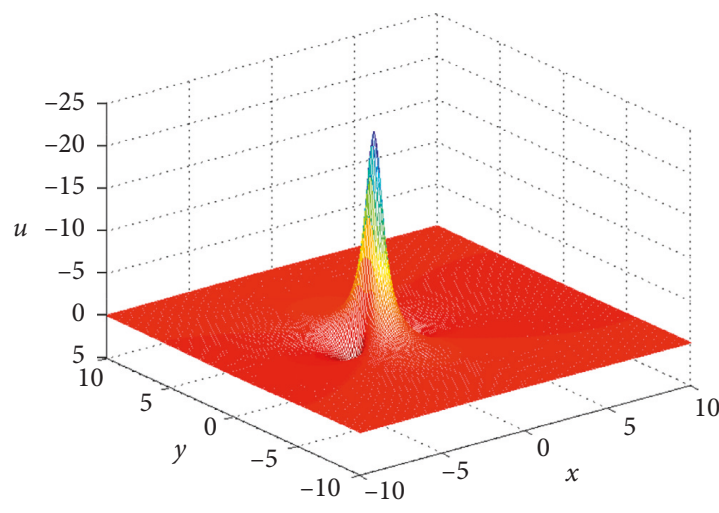

(a)

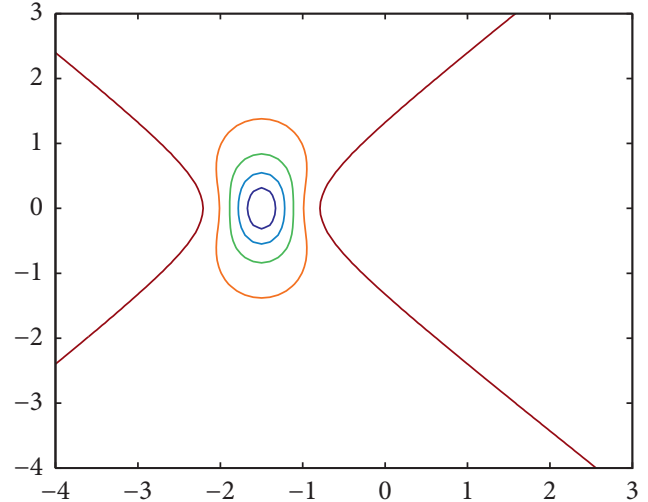

(b)

Figure 1: (a) Plots of lump solutions (8) with $\alpha_{1}=1, \alpha_{2}=1, \alpha_{4}=0, \alpha_{5}=1, \alpha_{8}=0, \alpha_{9}=1$, and $t=1$; (b) contour map in the ( $x, y$ ) plane.

(extreme value point); however, they have different extreme values (amplitudes) at the same minimum point. These two kinds of lump wave have the same mode of motion.

\section{Interaction Solutions Consisting of Lump Solutions and One Soliton Solution}

We will seek for the interaction solutions between lump solutions and one soliton solution. In order to do it, suppose that $F$ has the following form:

$$
\begin{aligned}
F & =f_{1}^{2}+f_{2}^{2}+l e^{\lambda}+\alpha_{9}, \\
f_{1} & =\alpha_{1} x+\alpha_{2} y+\alpha_{3} t+\alpha_{4}, \\
f_{2} & =\alpha_{5} x+\alpha_{6} y+\alpha_{7} t+\alpha_{8}, \\
\lambda & =\xi_{1} x+\xi_{2} y+\xi_{3} t,
\end{aligned}
$$

where $\alpha_{i}(1 \leq i \leq 9), l$, and $\xi_{i}(1 \leq i \leq 3)$ are real parameters to be determined.

Substituting equation (13) into equation (3) and considering the coefficients of all powers of the variables to be 0 , with the help of Maple, we have the following relations:

$$
\begin{aligned}
& \alpha_{3}=\frac{3}{2} \alpha_{1}, \\
& \alpha_{6}=-\frac{\alpha_{1} \alpha_{2}}{\alpha_{5}}, \\
& \alpha_{7}=\frac{3}{2} \alpha_{5}, \\
& \xi_{2}=0, \\
& \xi_{3}=-\xi_{1}\left(-\frac{3}{2}+\xi_{1}^{2}\right),
\end{aligned}
$$

where $\alpha_{1}, \alpha_{2}, \alpha_{4}, \alpha_{5}, \alpha_{8}, \alpha_{9}, l$, and $\xi_{1}$ are free parameters.

Thus, the interaction solutions between lump solutions and one soliton solution can be written as

$$
\begin{aligned}
u= & \left(-6\left[\left[( 2 ( \alpha _ { 1 } ^ { 2 } + \alpha _ { 5 } ^ { 2 } ) + l \xi _ { 1 } ^ { 2 } e ^ { \xi _ { 1 } x - \xi _ { 1 } ( - ( 3 / 2 ) + \xi _ { 1 } ^ { 2 } ) t } ) \left(\left(\alpha_{1} x+\alpha_{2} y+(3 / 2) \alpha_{1} t+\alpha_{4}\right)^{2}+\left(\alpha_{5} x-\left(\alpha_{1} \alpha_{2} / \alpha_{5}\right) y+(3 / 2) \alpha_{5} t+\alpha_{8}\right)^{2}+\alpha_{9}\right.\right.\right.\right. \\
& \left.\left.\left.\left.+l e^{\xi_{1} x-\xi_{1}\left(-(3 / 2)+\xi_{1}^{2}\right) t}\right)\right]-\left[2\left(\alpha_{1}\left(\alpha_{1} x+\alpha_{2} y+(3 / 2) \alpha_{1} t+\alpha_{4}\right)+\alpha_{5}\left(\alpha_{5} x-\left(\alpha_{1} \alpha_{2} / \alpha_{5}\right) y+(3 / 2) \alpha_{5} t+\alpha_{8}\right)\right)+l \xi_{1} e^{\xi_{1} x-\xi_{1}\left(-(3 / 2)+\xi_{1}^{2}\right) t}\right]^{2}\right]\right) \\
& \cdot\left(\left[\left(\alpha_{1} x+\alpha_{2} y+(3 / 2) \alpha_{1} t+\alpha_{4}\right)^{2}+\left(\alpha_{5} x-\left(\alpha_{1} \alpha_{2} / \alpha_{5}\right) y+(3 / 2) \alpha_{5} t+\alpha_{8}\right)^{2}+\alpha_{9}+l e^{\xi_{1} x-\xi_{1}\left(-(3 / 2)+\xi_{1}^{2}\right) t}\right]^{2}\right)^{-1} .
\end{aligned}
$$

Figure 3 shows the interaction solutions between lump solutions and one soliton solution. Similar to previous section, we know $F$ is analytical if and only if $\alpha_{5} \neq 0, \alpha_{9}>0$, and $l>0$. In order to facilitate dynamic analysis, we take $\alpha_{1}=1, \alpha_{2}=$ $1, \alpha_{4}=1, \alpha_{5}=1, \alpha_{8}=1, \alpha_{9}=1, \xi_{1}=1$, and $l=1$. When $t<-3$, there is only a solitary wave; at about $t=-2$, a special phenomenon occurs and the solitary wave starts to split into two parts: one is the lump wave and the other is the solitary wave. In this process, the amplitude of the lump wave has changed. When $t>-2$, the solitary wave still moves in the 


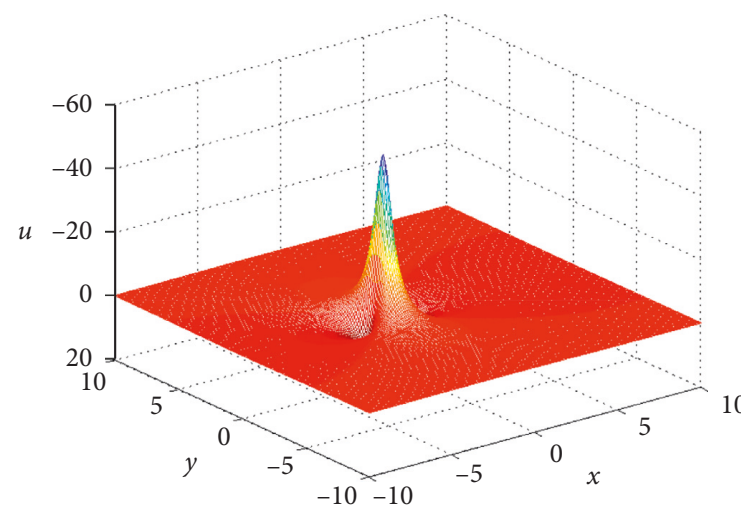

(a)

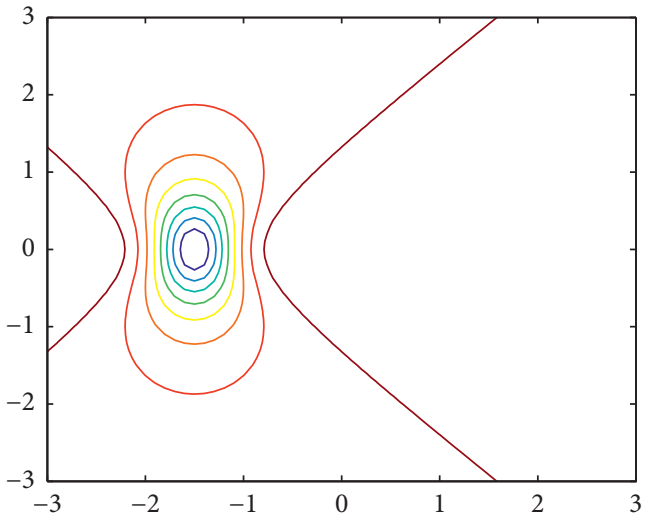

(b)

Figure 2: (a) Plots of lump solutions (12) with $\alpha_{2}=1, \alpha_{4}=0, \alpha_{6}=1, \alpha_{7}=1, \alpha_{8}=0, \alpha_{9}=1, \alpha_{10}=1, \alpha_{12}=0, \alpha_{13}=1$, and $t=1$; (b) contour map in the $(x, y)$ plane.

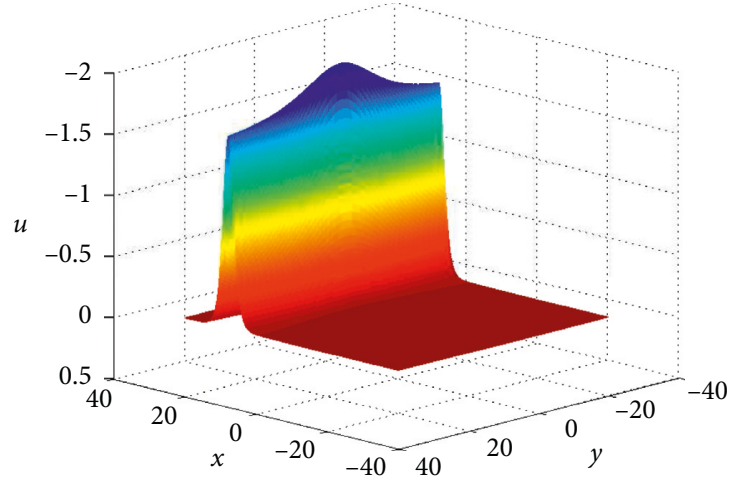

(a)

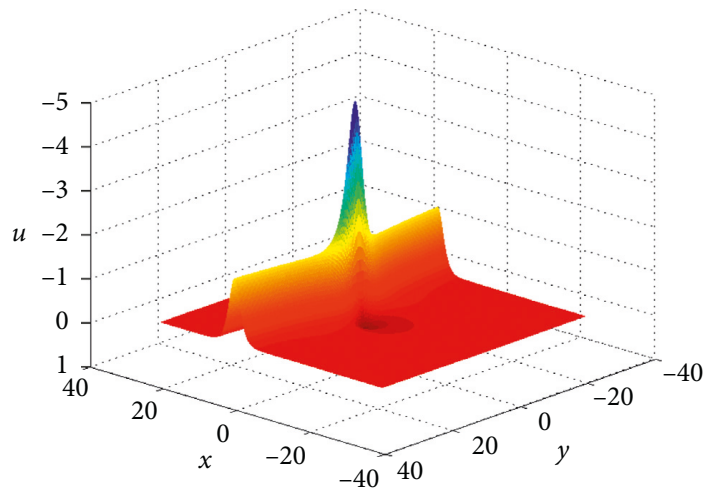

(c)

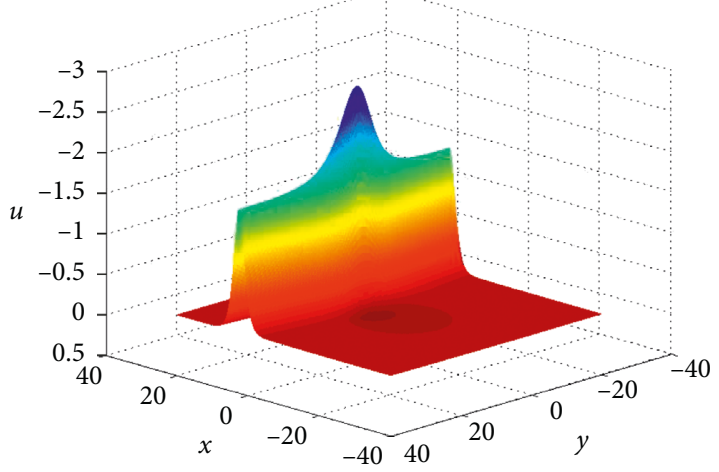

(b)

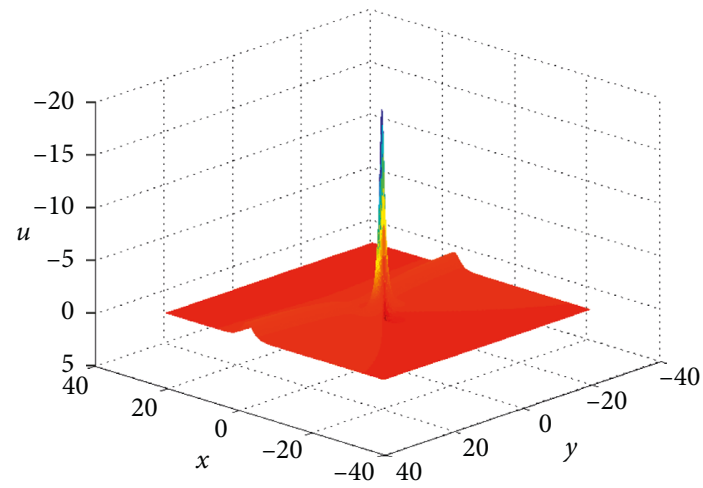

(d)

Figure 3: Continued. 


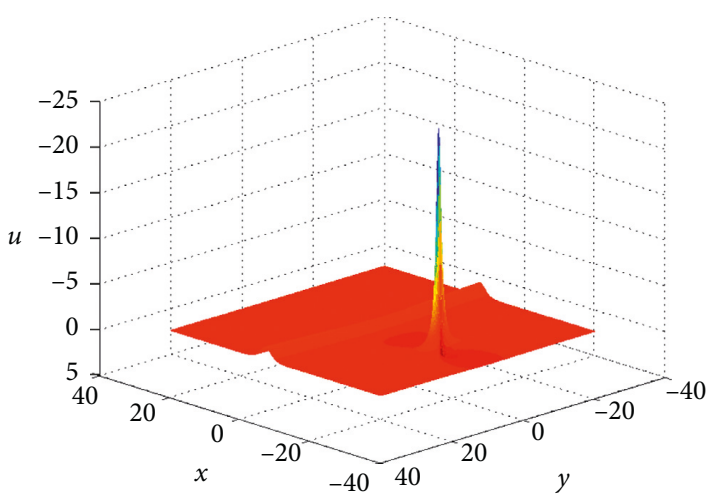

(e)

Figure 3: Fission phenomenon: plots of interaction solutions (15) with $\alpha_{1}=1, \alpha_{2}=1, \alpha_{4}=1, \alpha_{5}=1, \alpha_{8}=1, \alpha_{9}=1, \xi_{1}=1$, and $l=1$. (a) $t=-10$; (b) $t=-3$; (c) $t=-2$; (d) $t=0$; (e) $t=6$.

same direction as before, but the lump wave moves in the opposite direction, and they are farther and farther. This is a fission phenomenon. Figure 4 shows the opposite state of motion $\left(\xi_{1}=-1\right)$, where a lump wave and a solitary wave merge into a solitary wave. It is a fusion phenomenon. No matter fission or fusion, during the interaction, the solitary wave keeps its same amplitude and shape, and it is elastic. On the contrary, the lump wave's amplitude has changed, and it is inelastic.

\section{Interaction Solutions Consisting of Two Exponential Functions and One Sine Function}

In this section, we will seek for the interaction solutions consisting of two exponential functions and one sine function. In order to do it, suppose that $F$ has the following form:

$$
F=e^{-a_{1}\left(x+b_{1} y+c_{1} t\right)}+l_{1} e^{a_{1}\left(x+b_{1} y+c_{1} t\right)}+l_{2} \sin \left(a_{2}\left(x+b_{2} y+c_{2} t\right)\right.
$$

where $a_{1}, a_{2}, b_{1}, b_{2}, c_{1}, c_{2}, l_{1}$, and $l_{2}$ are parameters to be determined.

Substituting equation (16) into equation (3), with the help of Maple, we have the following relations:

$$
\begin{aligned}
& a_{1}=\frac{\sqrt{6}}{4}, \\
& a_{2}=\frac{\sqrt{-6}}{4}, \\
& b_{1}=b_{2}, \\
& c_{1}=c_{2}, \\
& l_{1}=\frac{l_{2}^{2}}{4},
\end{aligned}
$$

where $b_{2}, c_{2}$, and $l_{2}$ are free parameters.

For convenience, we let the parameters to be

$$
\begin{aligned}
& b_{2}=3, \\
& c_{2}=1, \\
& l_{2}=1 .
\end{aligned}
$$

Consequently, the interaction solutions consisting of two exponential functions and one sine function of equation (2) can be written as

$$
\begin{aligned}
u= & -6(\ln F)_{x x}=-6\left(\left[[ ( 3 / 8 ) e ^ { - ( \sqrt { 6 } / 4 ) ( x + 3 y + t ) } + ( 3 / 8 ) i \operatorname { s i n h } ( ( \sqrt { 6 } / 4 ) ( x + 3 y + t ) ) ] \cdot \left[e^{-(\sqrt{6} / 4)(x+3 y+t)}+(1 / 4) e^{\sqrt{6} / 4}\right.\right.\right. \\
& \left.\left.+i \sinh ((\sqrt{6} / 4)(x+3 y+t))]-\left[-(\sqrt{6} / 4) e^{-(\sqrt{6} / 4)(x+3 y+t)}+(\sqrt{6} / 4) i \cosh ((\sqrt{6} / 4)(x+3 y+t))\right]^{2}\right]\right) \\
& \cdot\left(\left[e^{-(\sqrt{6} / 4)(x+3 y+t)}+(1 / 4) e^{\sqrt{6} / 4}+i \sinh ((\sqrt{6} / 4)(x+3 y+t))\right]^{2}\right) .
\end{aligned}
$$

Figure 5 shows the interaction solutions consisting of two exponential functions and one sine function. Because $u$ is a complex solution, we plot its real part and imaginary part, respectively. From Figure 4(a), we find that the real part of $u$ is very similar to soliton solution. Its peaks are sharp while the soliton solution's peak is smooth. All of its peaks 


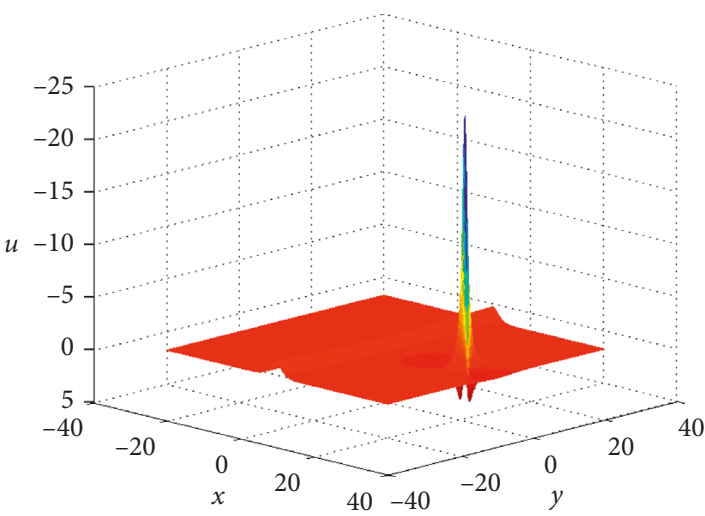

(a)

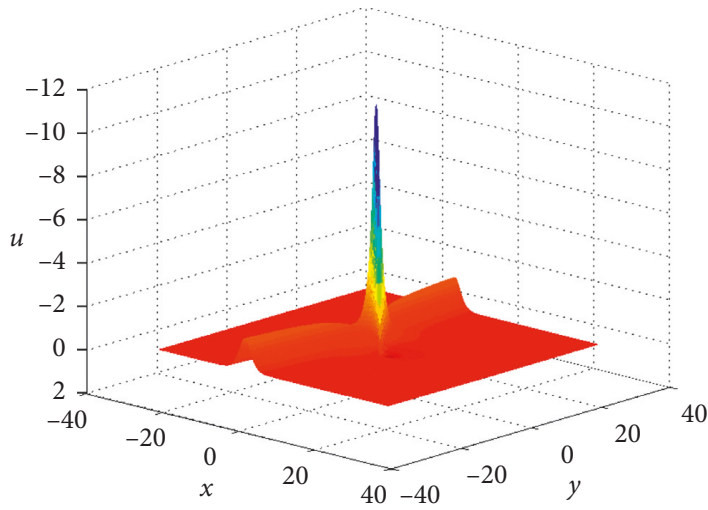

(c)

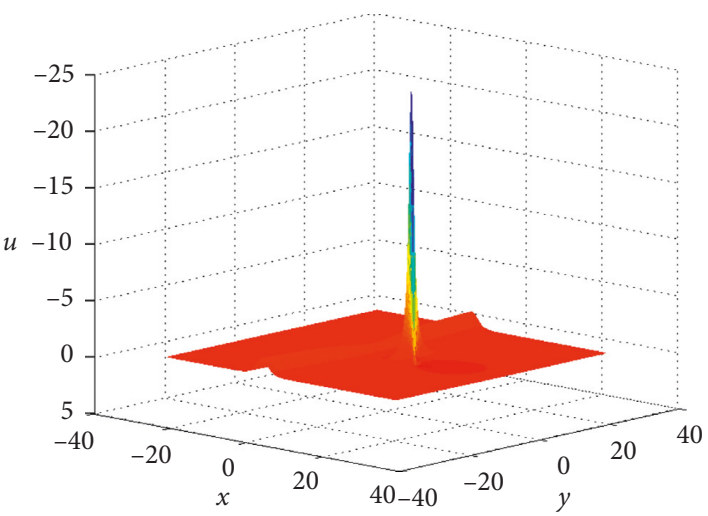

(b)

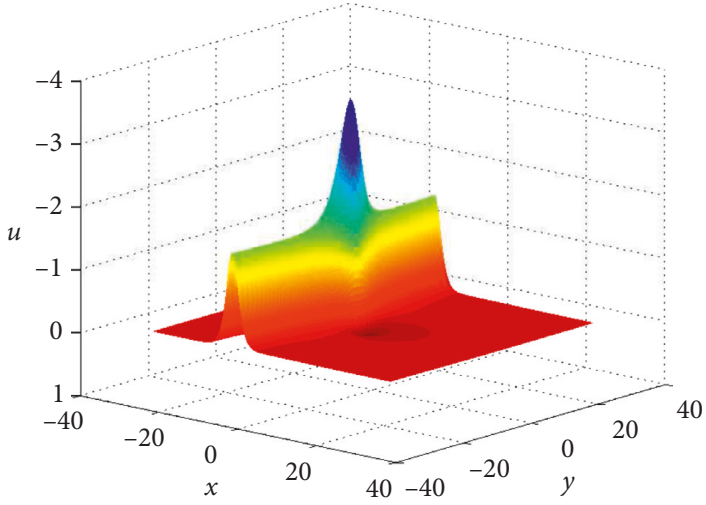

(d)

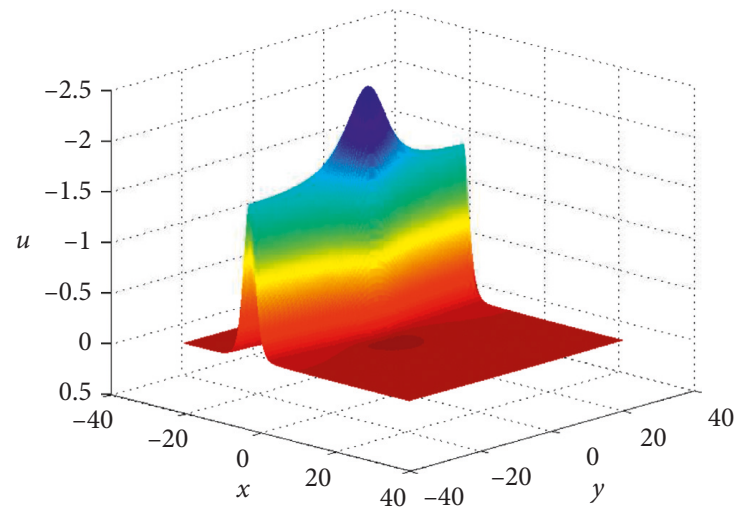

(e)

FIGURE 4: Fusion phenomenon: plots of interaction solutions (15) with $\alpha_{1}=1, \alpha_{2}=1, \alpha_{4}=1, \alpha_{5}=1, \alpha_{8}=1, \alpha_{9}=1, \xi_{1}=-1$, and $l=1$. (a) $t=-10$; (b) $t=-2$; (c) $t=0$; (d) $t=2$; (e) $t=6$.

have the same amplitude. From Figure 4(c), we can see the imaginary part of $u$ has two rows of peaks. All of its all peaks have the same amplitude, and this is similar to the real part. When $x$ or $y$ is given, the amplitude and shape of the real part of $u$ remain the same when $t$ changes, and this is similar to the imaginary part.

The obtained solution's real part has one row of sharp peaks, but its imaginary part has two rows of sharp peaks. As far as we know, such solutions with sharp peaks in both real and imaginary parts have rarely been seen in existing literatures.

\section{Conclusion}

As a summary, we investigate different kinds of solutions for a dimensionally reduced nonlinear evolution equation, including lump solutions and two kinds of interaction solutions. Dynamical characteristics and properties of obtained solutions are discussed, respectively. For these solutions, we discover many special physical phenomena, for example, the fission and fusion phenomena from the first kind of interaction solutions. The results show that the Hirota bilinear method is so prompt and effective to obtain solutions for 


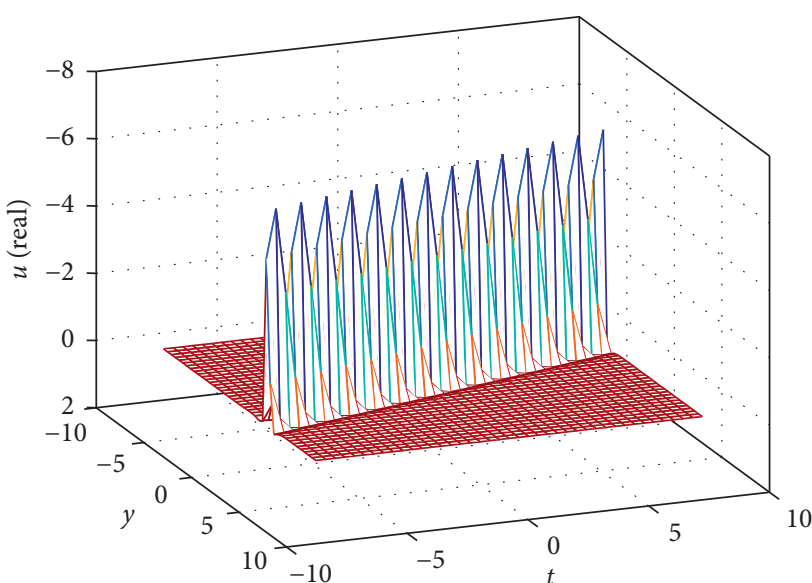

(a)

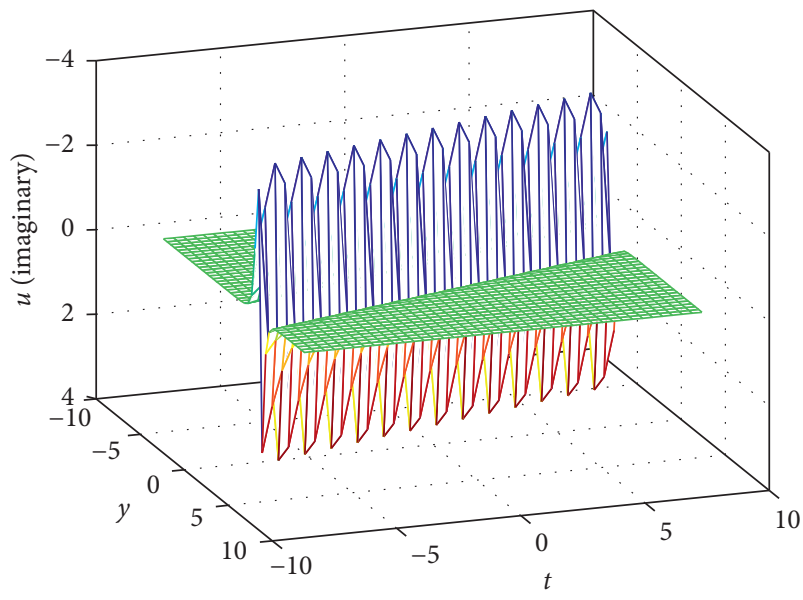

(c)

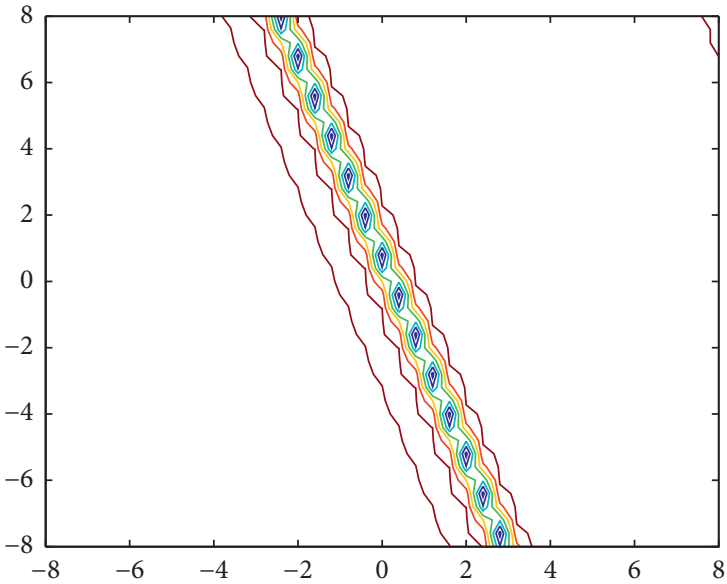

(b)

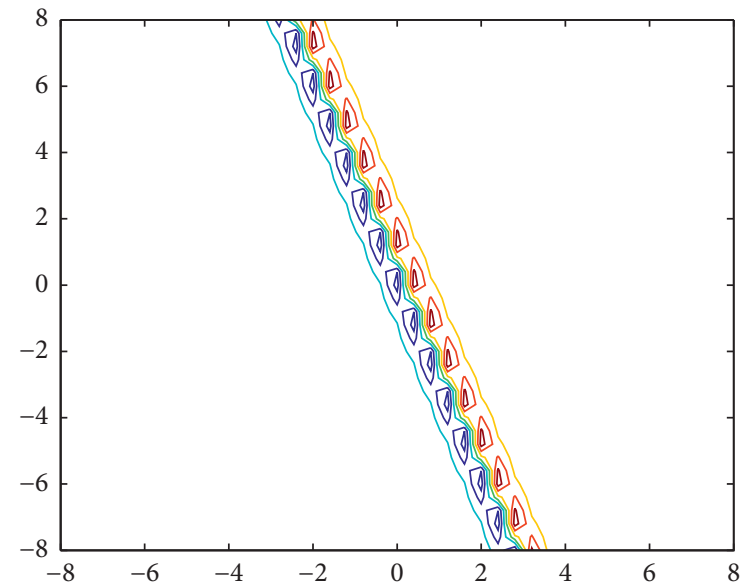

(d)

FIGURE 5: Plots of interaction solutions (19) with $b_{2}=3, c_{2}=1, l_{2}=1$, and $x=0$ : (a) the real part of $u$; (b) contour map of the real part; (c) the imaginary part of $u$; (d) contour map of the imaginary part.

nonlinear evolution equations. Based on this point, many other kinds of solutions for equations especially nonlinear mathematical physics equations are worth exploring.

\section{Data Availability}

No data were used to support this study.

\section{Conflicts of Interest}

The authors declare that they have no conflicts of interest.

\section{Acknowledgments}

This study was supported by the National Natural Science Foundation of China (grant nos. 11975143 and 61602188), Shandong Provincial Natural Science Foundation (grant no. ZR2019QD018), and Scientific Research Foundation of Shandong University of Science and Technology for Recruited Talents (grant nos. 2017RCJJ068 and 2017RCJJ069).

\section{References}

[1] W. X. Ma, Y. Zhang, Y. Tang, and J. Tu, "Hirota bilinear equations with linear subspaces of solutions," Applied Mathematics and Computation, vol. 218, no. 13, pp. 71747183, 2012.

[2] M. Russo and S. R. Choudhury, "Analytic solutions of a microstructure PDE and the KdV and Kadomtsev-Petviashvili equations by invariant Painlevé analysis and generalized Hirota techniques," Applied Mathematics and Computation, vol. 311, pp. 228-239, 2017.

[3] F. Yu and L. Li, "Inverse scattering transformation and soliton stability for a nonlinear Gross-Pitaevskii equation with external potentials," Applied Mathematics Letters, vol. 91, pp. 41-47, 2019.

[4] X. Zhang and Y. Chen, "Inverse scattering transformation for generalized nonlinear Schrödinger equation," Applied Mathematics Letters, vol. 98, pp. 306-313, 2019.

[5] M. S. Tao and H. H. Dong, " $N$-soliton solutions of the coupled kundu equations based on the Riemann-Hilbert method," Mathematical Problems in Engineering, vol. 2019, Article ID 3085367, 10 pages, 2019. 
[6] T. S. Liu and H. H. Dong, "The Prolongation structure of the modified nonlinear schrödinger equation and its initialboundary value problem on the half line via the RiemannHilbert approach," Mathematics, vol. 7, no. 2, p. 170, 2019.

[7] Y. X. Lin, Y. Fang, and H. H. Dong, "Prolongation structures and $N$-soliton solutions for a new nonlinear schrödinger-type equation via Riemann-Hilbert approach," Mathematical Problems in Engineering, vol. 2019, Article ID 4058041, 10 pages, 2019.

[8] A.-M. Wazwaz, "The tanh method and a variable separated ODE method for solving double sine-Gordon equation," Physics Letters A, vol. 350, no. 5-6, pp. 367-370, 2006.

[9] Y. Kong and Y. Fang, "On behavior of the correction equations in Jacobi-Davidson method," Mathematical Problems in Engineering, vol. 2019, Article ID 5169362, 4 pages, 2019.

[10] X. Lü, W.-X. Ma, and C. M. Khalique, "A direct bilinear Bäcklund transformation of a $(2+1)$-dimensional Kortewegde Vries-like model," Applied Mathematics Letters, vol. 50, pp. 37-42, 2015.

[11] Y. Fang, H. Dong, Y. Hou, and Y. Kong, "Frobenius integrable decompositions of nonlinear evolution equations with modified term," Applied Mathematics and Computation, vol. 226, pp. 435-440, 2014.

[12] H. Dong, Y. Fang, B. Guo, and Y. Liu, "Lie point symmetry, conservation laws and exact power series solutions to the Fujimoto-Watanabe equation," Quaestiones Mathematicae, pp. 1-17, 2019.

[13] W. X. Ma, "Lump solutions to the Kadomtsev-Petviashvili equation,” Physics Letters A, vol. 379, no. 36, pp. 1975-1978, 2015.

[14] Z. Ma, J. Chen, and J. Fei, "Lump and line soliton pairs to a (2+1)-dimensional integrable Kadomtsev-Petviashvili equation," Computers \& Mathematics with Applications, vol. 76, no. 5, pp. 1130-1138, 2018.

[15] Y. Zhang, H. Dong, X. Zhang, and H. Yang, "Rational solutions and lump solutions to the generalized $(3+1)$-dimensional shallow water-like equation," Computers \& Mathematics with Applications, vol. 73, no. 2, pp. 246-252, 2017.

[16] C. Wang and Z. Dai, "Breather-type multi-solitary waves to the Kadomtsev-Petviashvili equation with positive dispersion," Applied Mathematics and Computation, vol. 235, pp. 332-337, 2014.

[17] L.-L. Feng and T.-T. Zhang, "Breather wave, rogue wave and solitary wave solutions of a coupled nonlinear Schrödinger equation," Applied Mathematics Letters, vol. 78, pp. 133-140, 2018.

[18] R. Guo and H.-Q. Hao, "Breathers and multi-soliton solutions for the higher-order generalized nonlinear Schrödinger equation," Communications in Nonlinear Science and Numerical Simulation, vol. 18, no. 9, pp. 2426-2435, 2013.

[19] W. Liu and Y. Zhang, "Multiple rogue wave solutions for a (3+1)-dimensional Hirota bilinear equation," Applied Mathematics Letters, vol. 98, pp. 184-190, 2019.

[20] X. Zhang, Y. Chen, and X. Tang, "Rogue wave and a pair of resonance stripe solitons to KP equation," Computers \& Mathematics with Applications, vol. 76, no. 8, pp. 1938-1949, 2018.

[21] X. Wang, Y. Li, F. Huang, and Y. Chen, "Rogue wave solutions of AB system," Communications in Nonlinear Science and Numerical Simulation, vol. 20, no. 2, pp. 434-442, 2015.

[22] H. Wang, Y.-H. Wang, and H.-H. Dong, "Interaction solutions of a $(2+1)$-dimensional dispersive long wave system,"
Computers \& Mathematics with Applications, vol. 75, no. 8, pp. 2625-2628, 2018.

[23] B. S. Song and Y. Kong, "Solution to PSPACE-complete Problem using $\mathrm{P}$ systems with active membranes with timefreeness," Mathematical Problems in Engineering, vol. 2019, Article ID 5793234, 8 pages, 2019.

[24] J. Gu, Y. Zhang, and H. Dong, "Dynamic behaviors of interaction solutions of $(3+1)$-dimensional shallow water wave equation," Computers \& Mathematics with Applications, vol. 76, no. 6, pp. 1408-1419, 2018.

[25] W.-X. Ma, "Interaction solutions to the Hirota-Satsuma-Ito equation in $(2+1)$-dimensions," Frontiers of Mathematics in China, vol. 14, no. 3, pp. 619-629, 2019.

[26] W.-Q. Peng, S.-F. Tian, T.-T. Zhang, and Y. Fang, "Rational and semi-rational solutions of a nonlocal $(2+1)$-dimensional nonlinear Schrödinger equation," Mathematical Methods in the Applied Sciences, 2019.

[27] W.-X. Ma, "Application of the Riemann-Hilbert approach to the multicomponent AKNS integrable hierarchies," Nonlinear Analysis: Real World Applications, vol. 47, pp. 1-17, 2019.

[28] Y. Lin, H. Dong, and Y. Fang, "N-soliton solutions for the NLS-like equation and perturbation theory based on the Riemann-Hilbert Problem," Symmetry, vol. 11, no. 6, p. 826, 2019.

[29] W. X. Ma, "Riemann-Hilbert problems of a six-component mKdV system and its soliton solutions," Acta Mathematica Scientia, vol. 39B, pp. 509-523, 2019.

[30] W.-Q. Peng, S.-F. Tian, X.-B. Wang, T.-T. Zhang, and Y. Fang, "Riemann-Hilbert method and multi-soliton solutions for three-component coupled nonlinear Schrödinger equations," Journal of Geometry and Physics, vol. 146, Article ID 103508, 2019.

[31] W.-X. Ma, "Long-time asymptotics of a three-component coupled mKdV system," Mathematics, vol. 7, no. 7, p. 573, 2019.

[32] J. Chen, X. Hu, and S. Zhu, "Rational solutions of the $(2+1)-$ dimensional Kaup-Kupershmidt equation," Applied Mathematics Letters, vol. 95, pp. 150-157, 2019.

[33] L. Chen, J. Chen, and Q. Chen, "Mixed lump-soliton solutions to the two-dimensional Toda lattice equation via symbolic computation," Nonlinear Dynamics, vol. 96, no. 2, pp. 1531-1539, 2019.

[34] W.-X. Ma, J. Li, and C. M. Khalique, "A Study on lump solutions to a generalized Hirota-Satsuma-Ito equation in $(2+1)$-dimensions," Complexity, vol. 2018, Article ID 9059858, 7 pages, 2018.

[35] W. X. Ma, "A search for lump solutions to a combined fourthorder nonlinear PDE in $(2+1)$-dimensions," Journal of Applied Analysis and Computation, vol. 9, pp. 1319-1332, 2019.

[36] A.-M. Wazwaz, "A $(3+1)$-dimensional nonlinear evolution equation with multiple soliton solutions and multiple singular soliton solutions," Applied Mathematics and Computation, vol. 215, no. 4, pp. 1548-1552, 2009.

[37] W. X. Ma, "Bilinear equations, bell polynomials and linear superposition principle," Journal of Physics: Conference Series, vol. 411, Article ID 012021, 2013.

[38] H.-H. Dong and Y.-F. Zhang, "Exact Periodic wave solution of extended $(2+1)$-dimensional shallow water wave equation with generalized $D_{\bar{p}}$-operators," Communications in Theoretical Physics, vol. 63, no. 4, pp. 401-405, 2015. 


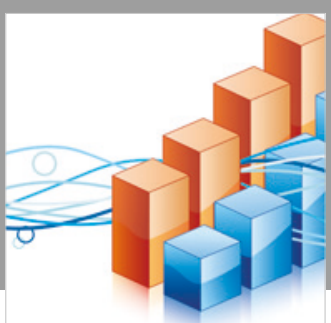

Advances in

Operations Research

\section{-n-m}
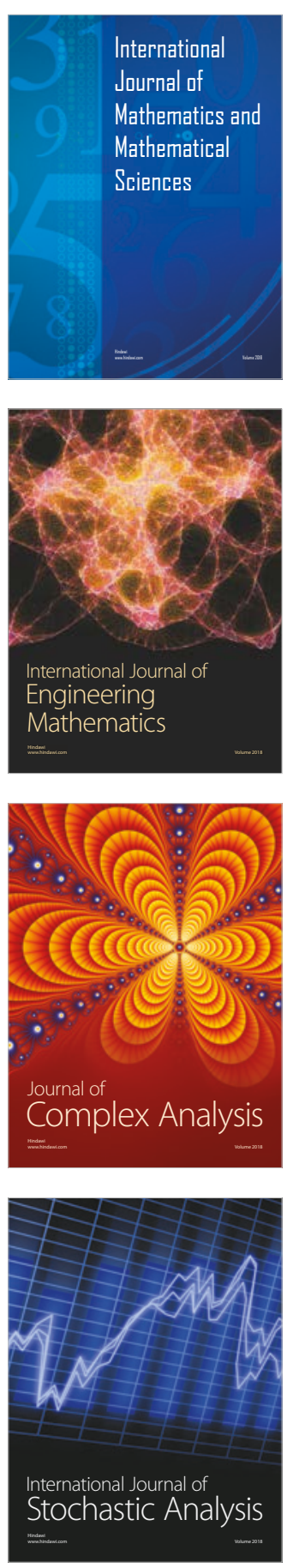
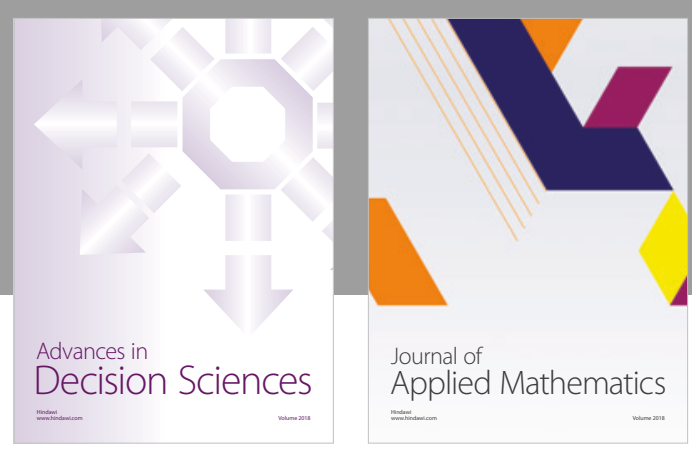

Journal of

Applied Mathematics
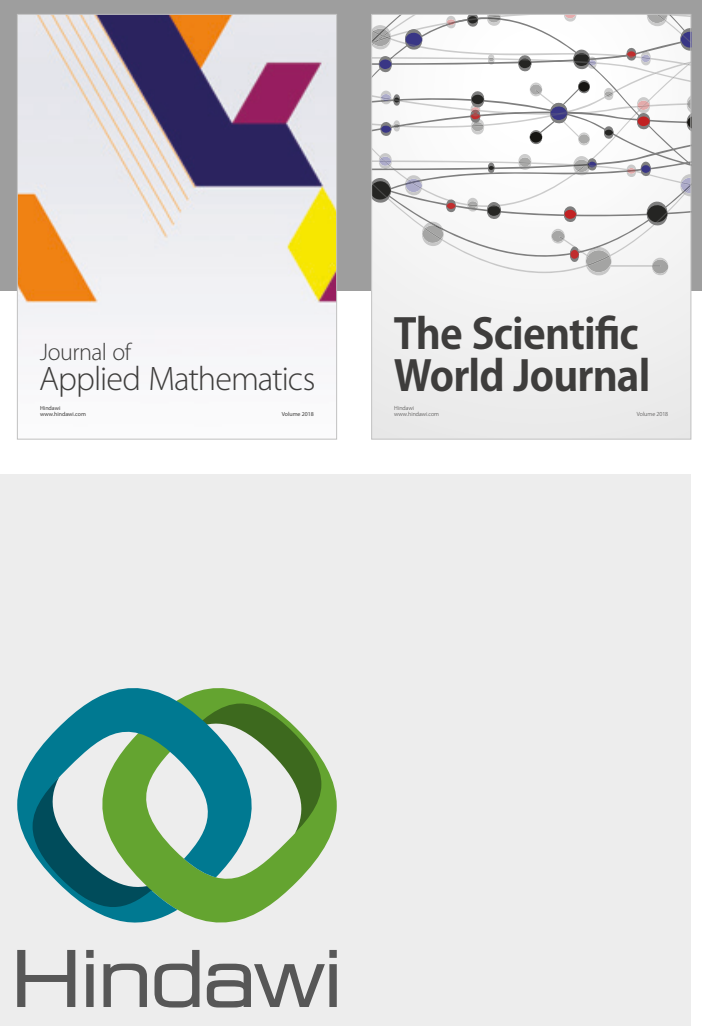

Submit your manuscripts at

www.hindawi.com

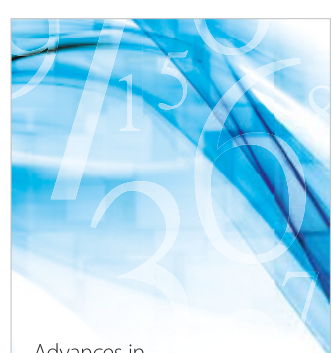

Advances in
Numerical Analysis
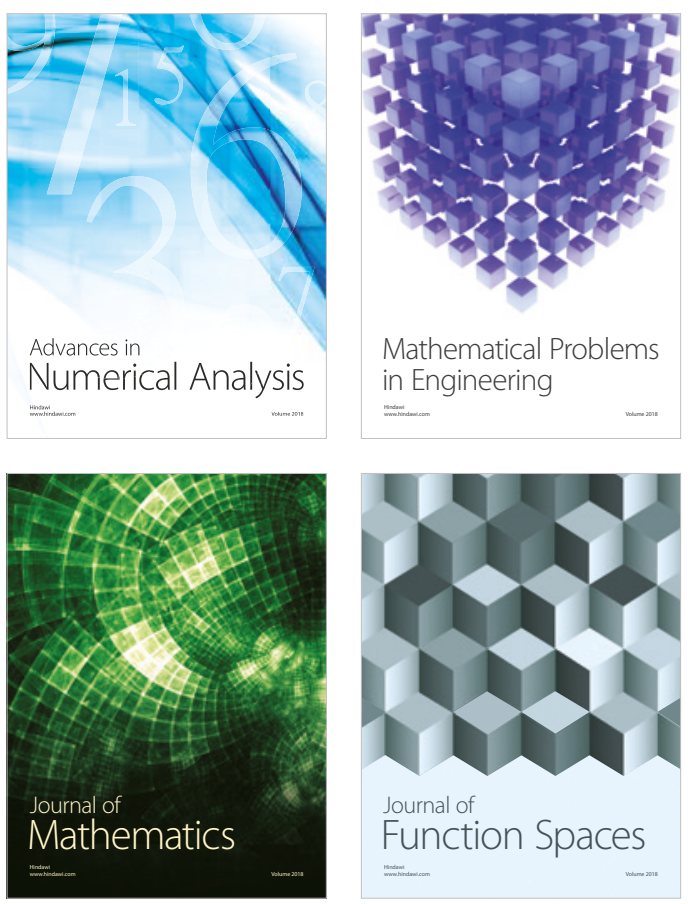

Mathematical Problems in Engineering

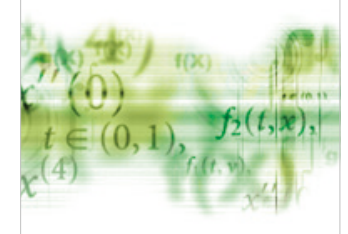

International Journal of

Differential Equations

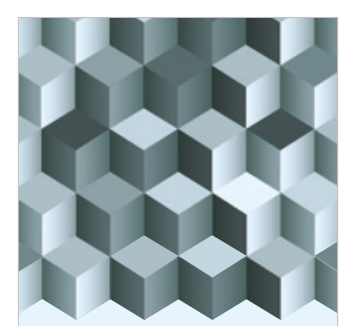

Journal of

Function Spaces
The Scientific

World Journal

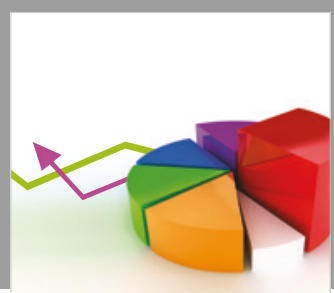

Journal of

Probability and Statistics
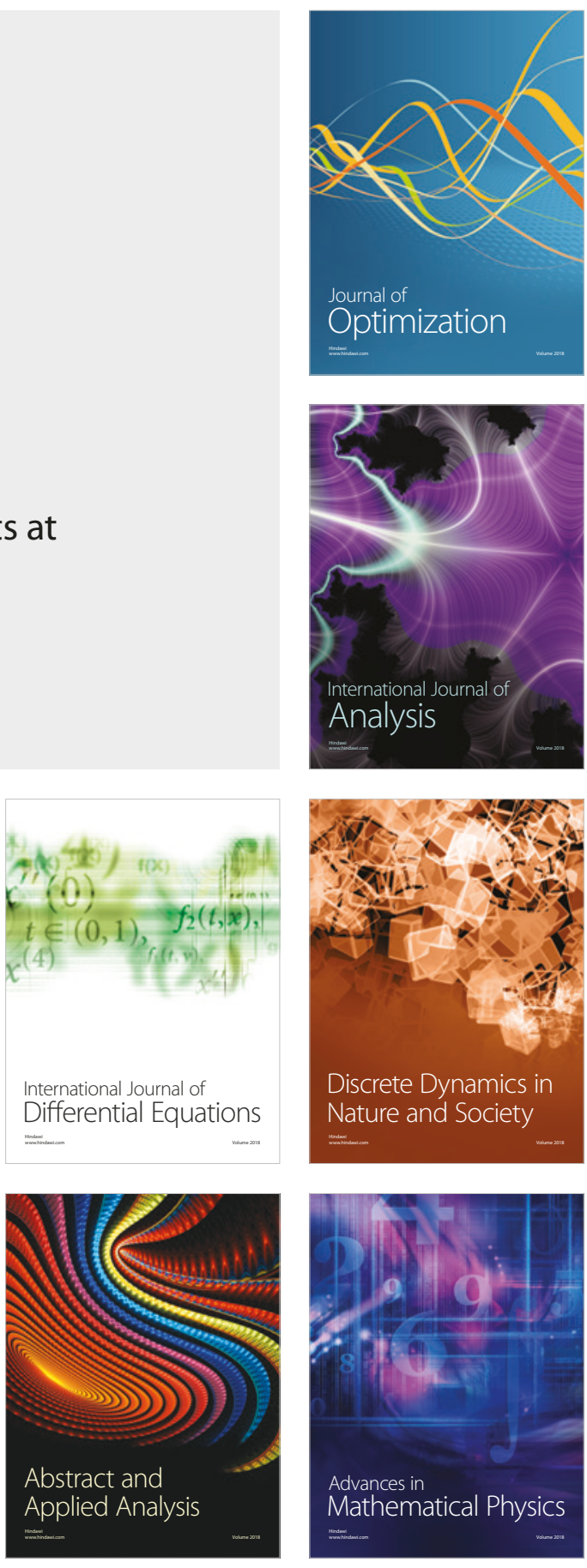